\title{
Guest-dependent negative thermal expansion in a lanthanide-based metal-organic framework
}

Received 00th January 20xx, Accepted 00th January 20xx

\author{
Andrzej Gładysiak, ${ }^{a}$ Seyed Mohamad Moosavi, ${ }^{a}$ Lev Sarkisov, ${ }^{\mathrm{b}}$ Berend Smit ${ }^{\mathrm{a}}$ and Kyriakos C. \\ Stylianou*a
}

\begin{abstract}
A lanthanide-based metal-organic framework (MOF), named SION2, displays strong and tuneable uniaxial negative thermal expansion (NTE). The coefficient of thermal expansion $\alpha_{1}$ reaches 153(6) $\mathrm{MK}^{-1}$ between 100 and $340 \mathrm{~K}$, which is among the highest values reported to date for $\mathrm{Ln}$-based MOFs. After heating to $380 \mathrm{~K}$ and subsequent cooling, the directionality and the magnitude of the effect change dramatically: the strong NTE effect is no longer observed along $X_{1}$, while a much weaker NTE effect emerges in a perpendicular direction. Loss of non-coordinated $\mathrm{N}, \mathrm{N}$ dimethylformamide molecules together with the rectuction of the size of structural voids stands as a rationale behind this switchover.
\end{abstract}

\section{Introduction}

Negative thermal expansion (NTE) is a counterintuitive property of certain materials that contract in at least one direction upon heating. ${ }^{1}$ Recently, a number of materials, including metal-organic frameworks (MOFs) exhibiting a wide range of NTE effects, have been reviewed. ${ }^{2}$ Aside from scientific curiosity and purely academic motivation to explain NTE phenomena on a fundamental level, interest in these materials is also driven by their potential applications in numerous fields where precise control over the thermal expansion is of supreme importance, i.e. electronic and optical devices as well as energyrelated technologies. ${ }^{3}$ In these applications, materials exhibiting NTE can be combined with much more common materials endowed with overall positive thermal expansion (PTE) to form a composite whose size over a given temperature range does not change; in other words, it displays zero thermal expansion (ZTE). ${ }^{4}$ Such approach minimises the temperature-induced strains, which may lead to improper performance or, in an extreme case, to an exfoliation and severe damage of the system.

There are numerous ways how the NTE effect is explained. Open-framework materials, in vast majority of cubic symmetry, derive their NTE from thermal population of low-energy

\footnotetext{
- Laboratory of Molecular Simulation (LSMO), Institut des Sciences et Ingénierie Chimiques (ISIC), École Polytechnique Fédérale de Lausanne (EPFL) Valais, Rue de I'Industrie 17, 1951 Sion, Switzerland.

b. Institute for Materials and Processes, School of Engineering, The University of Edinburgh, EH9 3J UK.

† Electronic Supplementary Information (ESI) available: experimental procedures, computational methods, additional crystallographic and structural data. See
} DOI: $10.1039 / x 0 \times x 00000 x$ transverse vibrational modes. $\mathrm{ZrW}_{2} \mathrm{O}_{8}$, an emblematic example of such frameworks, features a cubic lattice of metal ions $\mathrm{M}^{n+}$ interlinked by $\mathrm{O}^{2-}$ bridges. At increasing temperatures, the transverse vibrations of the $\mathrm{M}-\mathrm{O}-\mathrm{M}$ linkages bring the metal ions closer together within the lattice, resulting in an overall volumetric contraction, i.e. an NTE in three orthogonal directions. ${ }^{5}$ In many instances, the increased population of transverse vibrational modes is concomitant with distortions of edge-sharing polyhedra, as in the case of $\mathrm{ScF}_{3},{ }^{6} \mathrm{LiBeBO}_{3},{ }^{7}$ and $\mathrm{ReO}_{3}$-type compounds. ${ }^{8}$ Identical in terms of mechanism, but differing in magnitude (Table S1), the NTE effect was equally found in inorganic cyanides, $\mathrm{Zn}(\mathrm{CN})_{2}$ and $\mathrm{Cd}(\mathrm{CN})_{2},{ }^{9}$ nitrides, ${ }^{10}$ as well as in MOFs including HKUST-1,11 interpenetrated $\mathrm{Cu}_{3}(\mathrm{btb})_{2},{ }^{12} \mathrm{MOF}-5,{ }^{13}$ and MIL-68(In). ${ }^{14}$ In turn, graphite and $\mathrm{Ni}(\mathrm{CN})_{2}$ were reported to display a relatively rare biaxial NTE effect. ${ }^{15}$

In many instances, the NTE effect becomes more evident as a material approaches a phase transition. ${ }^{16} \mathrm{~A}$ typical example of such a behaviour is $\mathrm{PbTiO}_{3}$ perovskite, which exhibits increasingly stronger NTE effect near its ferroelectricparaelectric phase transition. ${ }^{17}$ NTE observed exclusively below the magnetic ordering temperature in $\mathrm{CuO}$ and $\mathrm{MnF}_{2}{ }^{18}$ is reminiscent of the Invar effect, i.e. virtual vanishing of thermal expansion in $\mathrm{Fe}-\mathrm{Ni}$ and $\mathrm{Fe}-\mathrm{Pt}$ alloys on approach towards the magnetic ordering temperature. ${ }^{19}$ Noteworthy is, however, that thermal expansion coefficients are in general temperaturedependent, and significant differences in their values as a function of temperature can be observed even if no phase transition takes place. ${ }^{20}$

Materials in which significant framework flexibility is responsible for the NTE constitute another large family. Most of these materials have symmetry lower than cubic, and exhibit NTE only in one specific direction, while the higher magnitude of PTE in two other directions gives rise to the overall positive volume thermal expansion. Inorganic framework materials (e.g., $\left.\mathrm{Ag}_{3}\left[\mathrm{Co}(\mathrm{CN})_{6}\right]\right),{ }^{21}$ molecular aggregates $\left(\mathrm{CD}_{3} \mathrm{OD} \cdot \mathrm{D}_{2} \mathrm{O}\right)^{22}$ and most importantly MOFs (HMOF-1, Zn(ISN) $2, \operatorname{lnD}(\mathrm{BDC})_{2}$, FJI-H11$\mathrm{R}, \mathrm{Zn}(\text { eim })_{2}$ and $\left.\mathrm{Cd}(\mathrm{eim})_{2}\right)^{23}$ owe their NTE to their intrinsic flexibility.

Thermal expansion behaviour of NTE materials can be influenced by the incorporation of guest molecules. ${ }^{24}$ The introduction of guest molecules can involve the change in magnitude of thermal expansion coefficient, ${ }^{25}$ reversing the sign thereof, $25 c, 25 d, 26$ or even the appearance of a new phase endowed with a markedly different thermal expansion. ${ }^{25 c,} 27$ 
Interesting examples of mechanisms leading to the guestdependent thermal expansion involve the substitution of a halide counterion, ${ }^{28}$ inserting helium into the structural cavities by means of hydrostatic pressure, ${ }^{29}$ or intercalation with lithium ions, $^{30}$ while adsorption of molecular species causing the change of the thermal expansion behaviour was predicted computationally. ${ }^{31}$

To further advance the field of materials exhibiting NTE, new strategies are required. An attractive suggestion comes from the field of coordination chemistry, where materials with diverse bonding topologies and chemistries show anomalous thermal and mechanical properties. ${ }^{32}$ In particular, while it is widely known that transition-metal complexes adopt only some discrete coordination numbers and preferred coordination geometries, lanthanide (Ln) ions, with their $4 \mathrm{f}$ orbitals buried inside the atomic core and well shielded by the filled $5 s$ and $5 p$ sub-shells, adopt a variety of coordination numbers and geometries, dominated by the steric effects of the ligands. ${ }^{33}$ Assembling such complexes into 3D polymers may give rise to a concerted response of the solid upon temperature increase, leading to anomalous thermal phenomena.

Herein, we combine the two approaches: (i) the assembly of a 3D framework and guest molecules together with (ii) the incorporation of Ln-atoms into a 3D structure, to develop a material endowed with a switchable NTE effect. In previous works, only the thermal expansion of Ln-based MOFs having charged particles (counterions) as guests was investigated, ${ }^{34}$ thus precluding the possibility of guest evacuation. The presently discussed Ln-based MOF, named SION-2, exhibits a high magnitude of NTE effect while it incorporates solvent guest molecules, however, once desolvated, it displays a significantly weaker NTE effect. The direction of NTE is also altered upon desolvation. Below we describe the origin of this effect studied with single-crystal X-ray diffractometry supported by molecular simulations.

\section{Experimental section}

SION-2 was crystallised following the previously reported procedure. ${ }^{35} \mathrm{~A}$ high-quality single crystal of SION-2 was isolated from the reaction mixture. It was mounted on a PILATUS@SNBL single-crystal X-ray diffractometer at the BM01 SwissNorwegian Beamline (ESRF, Grenoble, France). ${ }^{36}$ Preliminary exposures confirmed the singularity of the crystal. Reflection intensities were measured using the PILATUS2M detector. Raw data were processed with CrysAlisPro program suite, ${ }^{37}$ and the empirical absorption correction was performed using spherical harmonics, implemented in SCALE3 ABSPACK scaling algorithm. The structures were solved by the direct method and refined by the full-matrix least-squares method on $F^{2}$ with anisotropic thermal parameters for all non-hydrogen atoms. SHELXT and SHELXL programs, ${ }^{38}$ implemented in the Olex 2 program suite, ${ }^{39}$ were used for this purpose. Least-square refinement involved using the restraints on displacement parameters of some atoms. Positions of $\mathrm{H}$ atoms within the benzene rings and molecules of DMF were refined using the riding model, while $\mathrm{H}$ atoms within the hydroxyl groups were refined as in idealised rotating groups. $U_{\text {iso }}$ for non-terminal $\mathrm{H}$-atoms were set to 1.2 times $U_{e q}$ of neighbouring atoms, and for terminal $\mathrm{H}$-atoms 1.5 times $U_{\text {eq }}$ of neighbouring atoms. The experiment was initially performed at $100 \mathrm{~K}$, and then repeated in the 100-380 $\mathrm{K}$ temperature range at $20 \mathrm{~K}$ intervals on heating, and then in the 380-100 K temperature range on cooling. Selected crystallographic and structure refinement data for SION-2 can be found in Table S2 for the heating regime and in Table S3 for the cooling regime. In the $100-340 \mathrm{~K}$ temperature range, one non-coordinated DMF molecule was localised within the cavities of the main $\left[\mathrm{Tb}_{2}(\mathrm{DHBDC})_{3}(\mathrm{DMF})_{4}\right]$ framework; its disorder was refined as assuming two positions of inequivalent occupancy; moreover, displacement parameters and interatomic distances in both components of the disorder were constrained. In the 260-340 K temperature range, the disorder of one of the coordinated DMF molecules was also refined as assuming two positions of inequivalent occupancy. In the 360$380 \mathrm{~K}$ temperature range, the contribution of the heavily disordered DMF molecules found in the structural voids of SION-2 to the measured structure factors was quantified with the Olex 2 solvent masking procedure: ${ }^{40}$ at $360 \mathrm{~K}$ the integrated excess electron density of $27 \mathrm{e}^{-}$was found to correspond to $0.675 \mathrm{DMF}$ molecules per unit cell, while at $380 \mathrm{~K}$ the electron count equalled 0 . Coefficients of thermal expansion were calculated using the program PASCal (Principal Axis Strain Calculator). ${ }^{41}$ Topological simplification and net classification was performed using the TOPOS Pro program suite. ${ }^{42}$

\section{Computational methods}

Periodic density functional theory. Periodic density functional theory (DFT) calculations were performed using a plane wave basis set and projector augmented wave (PAW) pseudopotential using a plane-wave kinetic energy cutoff of 120 Ry as implemented in Quantum Espresso software package. ${ }^{43}$ The PBE exchange-correlation functional with DFTD2 dispersion correction were adopted in all calculations. The electronic structure was optimised using spin-polarised calculation by optimising the total magnetisation of the system. Each Tb"l has eight valence electrons in their forbitals. Two spin configurations were considered, namely a broken symmetry configuration with total magnetisation of zero, and a symmetric configuration with total magnetisation of twelve. The broken symmetry configuration with zero total magnetisation was found to be $41.2 \mathrm{kcal} / \mathrm{mol}$ more stable. The Brillouin-zone sampling was performed on gamma point only. The lattice parameters and atomic positions were relaxed by DFT, starting from the experimentally determined structure at $100 \mathrm{~K}$. Only the coordinated DMF molecules were kept, and the free DMF molecules residing within the pores were removed in order to facilitate the convergence to the minimum energy configuration. The input file for these calculations is included in Section S1.

Mechanical properties. The mechanical properties of the material were described with the fourth order stiffness tensor. The elements of the stiffness matrix were calculated based on 
the curvature of the potential energy surface of the material at the minimum energy configuration with respect to lattice deformations. We adopted a finite difference approach to compute the curvature of potential energy surface, using five sampled points per each lattice deformation with maximum strain rate of $1 \%$. An in-house code was used for generating the deformations and fitting the energy-strain curves. ${ }^{32 c}$ ELATE, a software package for stiffness tensor analysis, was used for all post processing and visualisation of the stiffness matrix. ${ }^{44}$

\section{Results and discussion}

SION-2, $\left[\mathrm{Tb}_{2}(\mathrm{DHBDC})_{3}(\mathrm{DMF})_{4}\right] \cdot 2 \mathrm{DMF} \quad\left(\mathrm{DHBDC}^{2-}=2,5-\right.$ dihydroxybenzene-1,4-dicarboxylate, DMF $=\mathrm{N}, \mathrm{N}$ dimethylformamide), crystallises in the triclinic space group $P \overline{1}$. Its framework is constructed upon $\mathrm{Tb}^{\mathrm{II}}{ }_{2}$ binuclear clusters interlinked through three types of $\mathrm{DHBDC}^{2-}$ ligands: one $\eta^{2}$ chelating and two distinct $\eta^{1}: \eta^{1}$ bridging ligands. The $\mathrm{Tb}^{1 \mathrm{II}_{2}}$ clusters and all types of DHBDC ${ }^{2-}$ ligands are centrosymmetric as they lie about inversion centres. The DMF molecules coordinated to the Tb"II metal centres, and those occupying the structural voids (guest molecules), are present in the structure of SION-2 in a ratio of 2:1. Tb"II atoms are 8-coordinated and display a square antiprismatic coordination geometry. SION-2 has recently been assigned to the $\boldsymbol{x a h}$ net topology, ${ }^{35}$ but it can be further simplified to the $\alpha$-Po type, pcu net (Figs. 1 and S1) by regarding the $\mathrm{Tb}^{111}{ }_{2}$ clusters as a single node rather than as two separate nodes.

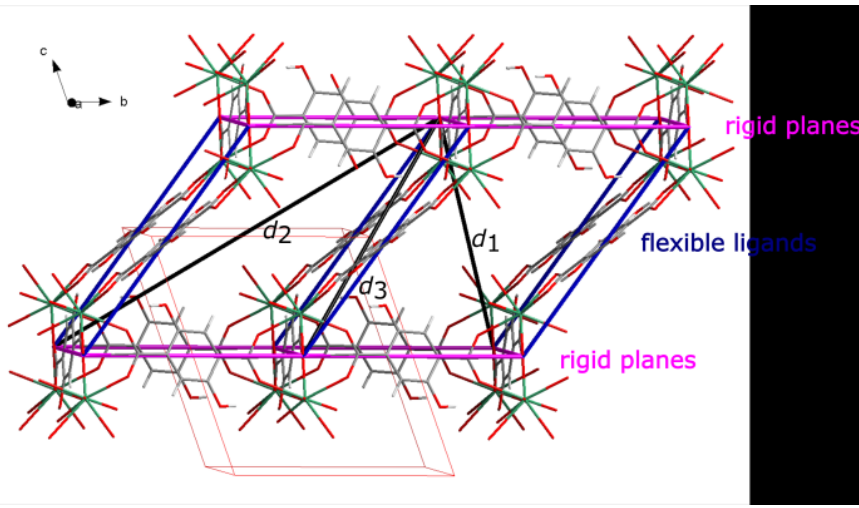

Fig. 1 Crystal structure of SION-2 and the contour of its unit cell outlined with the nodes of the underlying pcu net (centres of the $\mathrm{Tb}^{\mathrm{III}}{ }_{2}$ clusters) overlaid. Distances $d_{1}, d_{2}$, and $d_{3}$ are a function of the unit-cell parameters. DMF molecules have been omitted for clarity.

The thermal expansion of SION-2 was studied with variabletemperature single-crystal X-ray diffractometry (VT SCXRD) using synchrotron radiation. While heated in the $100-340 \mathrm{~K}$ temperature range, SION-2 experiences a continuous unit-cell volume expansion with no phase transitions (Table S4). The thermal expansion of SION-2 is heavily anisotropic with the 6 and $c$ parameters expanding to the largest extent, and at $340 \mathrm{~K}$ equalling respectively $104.3 \%$ and $103.6 \%$ of their initial values. The thermal expansion coefficients $\alpha_{\ell}=(1 / \ell) \mathrm{d} \ell / \mathrm{d} T$ calculated with respect to the three principal axes $X_{1}, X_{2}$, and $X_{3},{ }^{45}$ revealed an NTE along $X_{1}$, a moderate PTE along $X_{2}$, and a significant PTE along $X_{3}$ (Tables 1 and S5, Fig. S2). SION-2 exhibits NTE along $X_{1}$ with $\alpha_{1}=-153(6) \mathrm{MK}^{-1}$, which is the highest value among all lanthanide-based MOFs reported to date. Ammonium metal formate framework $\left[\mathrm{NH}_{2} \mathrm{NH}_{3}\right]\left[\mathrm{Tm}(\mathrm{HCOO})_{4}\right]$ was shown to display a slightly weaker NTE effect of $\alpha_{3}=-120 \mathrm{MK}^{-1}$ over a narrower $100-180 \mathrm{~K}$ temperature range, ${ }^{34 a}$ while the effect in $\left[\mathrm{NH}_{2} \mathrm{CHNH}_{2}\right]\left[\mathrm{Er}(\mathrm{HCOO})_{4}\right]$ was weaker by one order of magnitude $\left(\alpha_{2}=-7 \mathrm{MK}^{-1}\right)$ in the $60-300 \mathrm{~K}$ temperature range. ${ }^{34 \mathrm{~b}}$

The principal axes $X_{1}, X_{2}$, and $X_{3}$ nearly coincide with the face diagonals of parallelepipeds forming the primitive $\alpha$-Po net. $d_{1}$ is defined as the shorter diagonal of the parallelogram formed by two $\eta^{2}$-chelating and two O2,O4-bridging DHBDC ${ }^{2-}$ ligands, $d_{2}$ as the longer diagonal of this parallelogram, and $d_{3}$ as the shorter diagonal of the parallelogram formed by two $\eta^{2}$ chelating and two 03,06-bridging DHBDC ${ }^{2-}$ ligands (Figs. 1 and $\mathrm{S} 1$; the $\mathrm{O}$ atom numbering refers to the scheme in the originally published crystal structure). Due to the fact that the nodes of the pcu net occupy special $1 g$ positions of the triclinic unit cell $(0,1 / 2,1 / 2)$, the lengths of $d_{1}, d_{2}$, and $d_{3}$ can be directly inferred from the adequate unit-cell parameters (Fig. S3). The change of these distances as a function of temperature is presented in Fig. S4. The distance $d_{1}$ shortens to a significant extent, while $d_{2}$ increases slightly, and $d_{3}$ increases by $0.77 \AA$ in the $100-340 \mathrm{~K}$ temperature range. Expressing the progression of these distances in the percentage scale (Fig. 2b) emphasises the same temperature-induced trends as observed in the case of principal axis lengths (Fig. 2a). For the purpose of comparison, the coefficients of thermal expansion of the distances $d_{1}, d_{2}$, and $d_{3}$ were also calculated, and reported in Table 1 . During the heating run, in the 100-340 $\mathrm{K}$ temperature range, they reflect the same trends as the coefficients derived along the principal axes. During the subsequent cooling, in the 340-100 K temperature range, however, the change of $d_{1}, d_{2}$, and $d_{3}$ with temperature is markedly different. The most significant, yet lower in value, PTE is observed along $d_{3}, d_{1}$ displays a moderate PTE, while weak NTE is observed along $d_{2}$.

Geometrically, SION-2 can be considered as constructed by planes of $\mathrm{Tb}^{111}{ }_{2}$ clusters interconnected with 02,04- and 03,06bridging $\mathrm{DHBDC}^{2-}$ ligands (horizontal layers in Fig. 1). Multiple coordination bonds constituting these planes $(\mathrm{O}$ atoms of carboxylate groups of DHBDC ${ }^{2-}$ bind simultaneously to two $\mathrm{Tb}^{\prime \prime \prime}$ atoms within a $\mathrm{Tb}^{\prime \prime \prime} 2$ unit) should in theory provide the planes with a certain inflexibility upon temperature increase as any potential change of position of one $\mathrm{O}$ atom in the coordination shell of the first Tb"II atom ought to involve a concerted change of the second O coordinated to the neighbouring Tb"' (Fig. S5a). On the other hand, the Tb"I-bridging $\mathrm{DHBDC}^{2-}$ planes are linked together with chelating $\mathrm{DHBDC}^{2-}$ ligands; they coordinate to the same $\mathrm{Tb}^{\text {III }}$ with both carboxylate $\mathrm{O}$ atoms (in a manner similar to a ball-and-socket linkage endowed with a considerable rotational freedom), so change of their position with respect to the $\mathrm{Tb}^{\mathrm{III}}{ }_{2}$ cluster should not be constrained (Fig. S5b). 
a

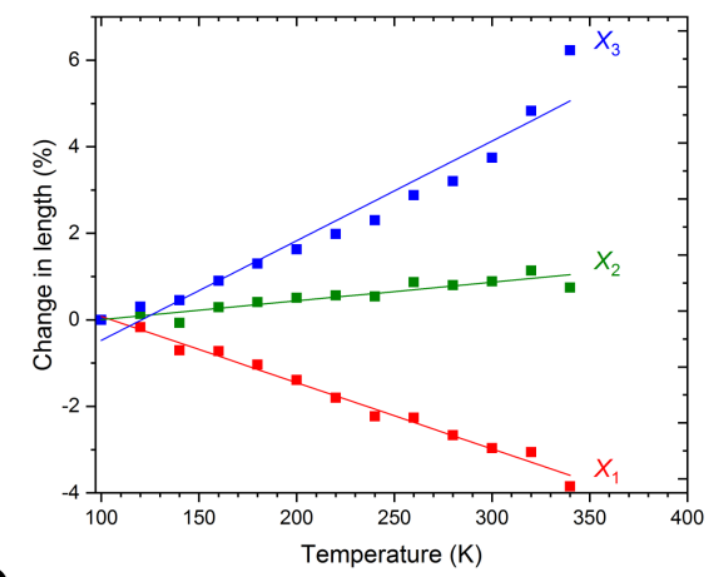

b

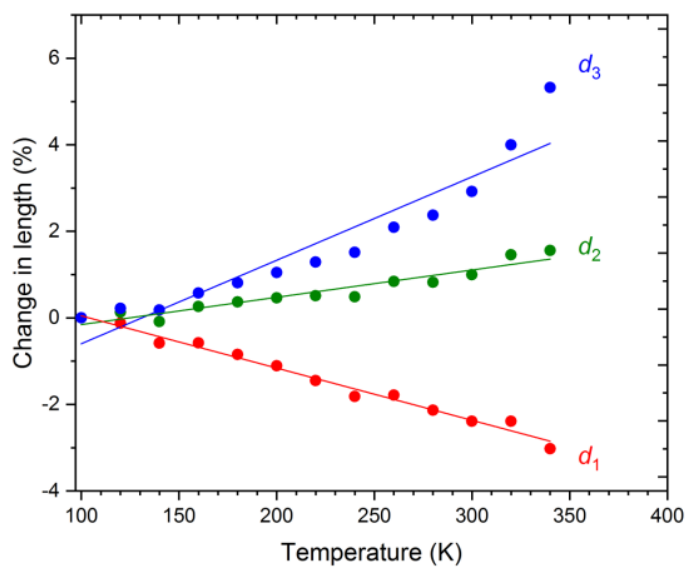

C

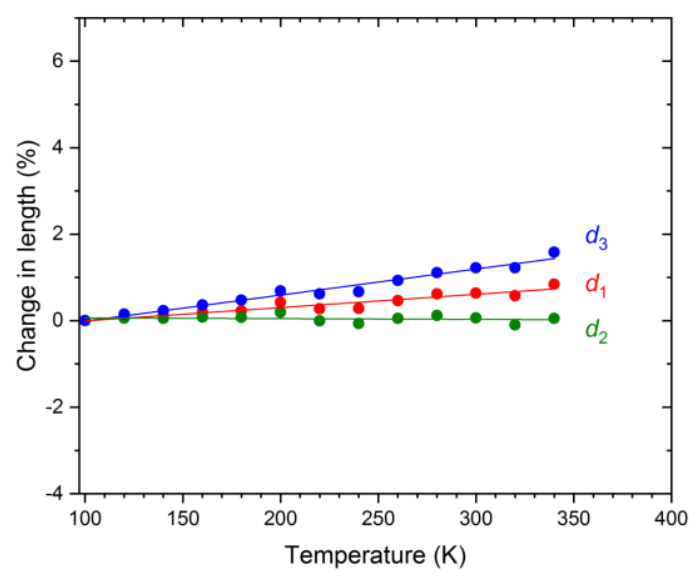

Fig. 2 (a) Percentage change in lengths of the principal axes $X_{1}$, $X_{2}$, and $X_{3}$ of SION-2 as a function of temperature. (b) Percentage change in distances $d_{1}, d_{2}$, and $d_{3}$ in the $100-340 \mathrm{~K}$ temperature range on heating. (c) Percentage change in distances $d_{1}, d_{2}$, and $d_{3}$ in the 340-100 $\mathrm{K}$ temperature range on cooling. The coefficients $\alpha$ of thermal expansion (Table 1 ) are effectively the average slopes of these curves marked with solid lines.
Table 1 Coefficients $\alpha$ of thermal expansion determined experimentally along three principal axes $X_{1}, X_{2}$, and $X_{3}$ in the 100-340 $\mathrm{K}$ temperature range during the heating regime, and coefficients $\alpha$ of thermal expansion of the distances $d_{1}, d_{2}$, and $d_{3}$ in the 100-340 $\mathrm{K}$ temperature range during the heating and cooling regimes.

\begin{tabular}{cccc}
\hline \multicolumn{4}{c}{$100-340 \mathrm{~K}$, heating } \\
Axis & $\alpha\left(\mathrm{MK}^{-1}\right)$ & Distance & $\alpha\left(\mathrm{MK}^{-1}\right)$ \\
\hline$X_{1}$ & $-153(6)$ & $d_{1}$ & $-121(4)$ \\
$X_{2}$ & $+43(6)$ & $d_{2}$ & $+63(5)$ \\
$X_{3}$ & $+230(20)$ & $d_{3}$ & $+190(20)$ \\
\hline \multicolumn{4}{c}{$340-100 \mathrm{~K}$, cooling } \\
& Distance & $\alpha\left(\mathrm{MK}^{-1}\right)$ \\
\hline & $d_{1}$ & $+32(2)$ \\
& $d_{2}$ & $-4(2)$ \\
& $d_{3}$ & $+61(2)$ \\
\hline
\end{tabular}

To provide more insights, VT SCXRD measurements were further analysed with full-matrix least-squares refinement. Derivation of specific conformational parameters helped elucidate the NTE effect in many previous cases. ${ }^{14,23 c, 23 d, 46}$ In SION-2, as the temperature is increased, the Tb"II-bridging DHBDC $^{2-}$ planes slide on the top of each other while the angle between the chelating $\mathrm{DHBDC}^{2-}$ and the planes decreases (Fig. S6). In particular, the slide is realised in such a way that the distance $d_{1}$ shortens considerably, and the distance $d_{2}$ lengthens as the temperature is increased, leading to respectively NTE along $d_{1}$ and PTE along $d_{2}$. This phenomenon is clearly visible in the Supplementary Videos 1 and 2 depicting the change of the structure as a function of temperature as viewed along [100] and [010], respectively (in both cases the rigid planes are lined in horizontal position). It is highly probable that in an extreme case, the slide of the Tb"II-bridging $\mathrm{DHBDC}^{2-}$ planes may lead to a mutual connection of $\mathrm{Tb}^{\prime{ }^{\prime \prime}}{ }_{2}$ clusters into $1 \mathrm{D}$ $\mathrm{Tb}-\mathrm{O}$ chains. The chemical transformation of SION-2 into another Tb-based MOF, [Tb 2 (DHBDC)(DOBDC)(DMF) 2 ], (SION-1, where $\mathrm{DOBDC}^{-}=$2,5-dioxidobenzene-1,4-dicarboxylate), which realises this scenario, was reported previously. ${ }^{35}$

The least-square refinement also provides insights about the structural changes occurring at $380 \mathrm{~K}$. A decrease of $12.7 \%$ of the unit-cell volume in the 360-380 K temperature range (Fig. $\mathrm{S} 7 \mathrm{a}, \mathrm{b}$ and Table $\mathrm{S} 4$ ) is accompanied by the significant reduction of the void volume (from $17.7 \%$ of the unit-cell volume at $360 \mathrm{~K}$ to $12.7 \%$ at $380 \mathrm{~K}, \mathrm{Fig}$. S8) and content (at $360 \mathrm{~K}$, noncoordinated DMF molecules become highly disordered, contributing to the diffuse electron density corresponding to $0.675 \mathrm{DMF}$ molecules per unit cell, and at $380 \mathrm{~K}$ they vanish, Fig. S9). The evacuation of solvent molecules is in line with the weight loss observed from the thermogravimetric analysis reported previously. ${ }^{35}$ Once desolvated, the crystal of SION-2 exhibits a strikingly different thermal expansion pattern. The slide of the rigid $\mathrm{Tb}^{\text {III-}}$-bridging DHBDC ${ }^{2-}$ planes is reversed (Fig. S6), however, the geometry of this slide is different compared 
to the heating run. Two closest $\mathrm{Tb}^{\mathrm{II}}{ }_{2}$ cluster originating from two subsequent rigid planes come closer to each other as the temperature is lowered, which results in PTE along $d_{1}$. In turn, the distance $d_{2}$ lengthens on cooling, which is reflected by a small, yet definitely negative coefficient of thermal expansion of $d_{2}$ (Supplementary Video 3 ). In the direction perpendicular to both $d_{1}$ and $d_{2}$, the result of heating is analogous to that of cooling, since during both temperature ramps the strongest PTE is observed along $d_{3}$.

To cross-check our observations of NTE and PTE in SION-2 and to validate the postulated mechanism, its mechanical properties were studied computationally. A simplified structure of SION-2, wherein the free non-coordinated DMF molecules residing in the pores had been removed, was used in the computations for the purpose of easier convergence to the minimum energy. Therefore, these computations correspond to the experiment in the 380-100 K temperature range on cooling, when the crystal was found not to comprise guest DMF molecules. The stiffness matrix, whose interrelation to the thermal expansion of materials has widely been known, ${ }^{47}$ was computed for SION-2 using density functional theory (DFT). This matrix, which describes the stress-strain relation for a crystal in the elastic regime, is symmetric, and its 21 elements are independent for the triclinic SION-2.45 The mechanical properties of the material are summarised in Table S7, while the $3 \mathrm{D}$ representation of the Young's modulus computed based on the stiffness matrix is shown in Fig. 3a. High maxima of the Young's modulus of $15 \mathrm{GPa}$ (Fig. 3a) are indicative of its relative stiffness in directions parallel to the Tb"ll-bridging DHBDC ${ }^{2-}$ planes. In turn, the value of Young's modulus drops to $0.15 \mathrm{GPa}$ and $0.4 \mathrm{GPa}$ along two soft modes of the crystal, suggesting that these rigid planes can relatively freely move on top of each other due to the weak linkage between them.

Interestingly, whenever the crystal of SION-2 is kept in a relatively low-temperature regime (not higher than $300 \mathrm{~K}$ ), the changes in unit-cell parameters are quasi-reversible (Fig. S7c,d and Table S8). Although the changes in principal axes lengths do not follow exactly the same scheme upon cooling and heating in the 100-300-100 K temperature range, the unit-cell volume change shows no hysteresis. This observation emphasises the impact of presence or absence of guest DMF molecules on the thermal expansion coefficients of SION-2.

The observation of the NTE effect in SION-2 strongly suggests a possibility of occurrence of another structural effect, namely negative linear compressibility (NLC), which designates a situation whereby a hydrostatically compressed crystal expands in one particular direction. These two phenomena are independent one from another as there is no thermodynamic requirement that NLC and NTE must coexist. ${ }^{48}$ Nevertheless, NTE and NLC effects were previously observed experimentally in a range of materials, ${ }^{21-22,} 49$ although the magnitude, directionality and underlying mechanisms of NTE and NLC were shown to differ one from another in some of those materials. ${ }^{24,}$ 25b, 50 Noteworthy is, however, that the experimental quantification of NLC in crystals with triclinic symmetry, such as SION-2, present a considerable challenge. Therefore, we resorted to computational methods in order to shed light on this matter. As represented in Fig. 3b, we expect SION-2 to exhibit a biaxial NLC with a minimum of $-284 \mathrm{TPa}^{-1}$ and a uniaxial positive linear compressibility with a maximum of +605 $\mathrm{TPa}^{-1}$. a

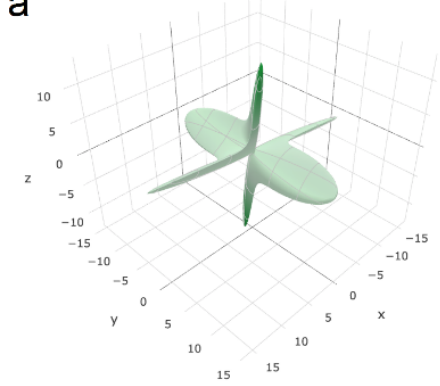

b

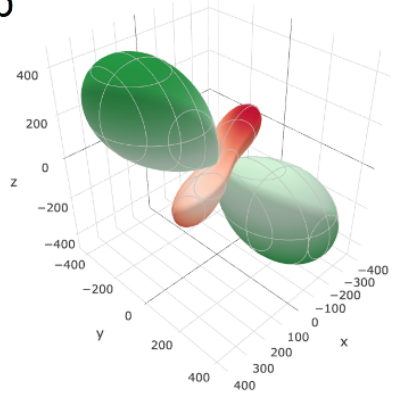

Fig. 3 The 3D representation of (a) Young's modulus, and (b) linear compressibility calculated for SION-2 with noncoordinated DMF molecules removed from the pores. Values of Young's modulus and linear compressibility are in GPa and TPa${ }^{1}$ respectively.

\section{Conclusions}

Thermal expansion of a Ln-based MOF having labile solvent guest molecules within the structural voids was experimentally and computationally studied for the first time. When heated in the $100-340 \mathrm{~K}$ temperature range, SION-2 exhibits a uniaxial negative thermal expansion along $X_{1}$ with $\alpha_{1}=-153(6) \mathrm{MK}^{-1}$, which manifests itself as a slide of rigid Tb"II-bridging DHBDC $^{2-}$ planes interconnected with mobile chelating DHBDC ${ }^{2-}$ ligands on the top of each other, as observed from the VT SCXRD measurements. This phenomenon, however, occurs only when the DMF guest molecules are present within the structural voids. Once heated up to $380 \mathrm{~K}$, the crystal loses all noncoordinated solvent, which is accompanied by a significant reduction of the void size. Upon subsequent cooling, the thermal expansion of the crystal is markedly different. The slide of the rigid Tb"I-bridging DHBDC ${ }^{2-}$ planes is reversed, however, the progression of $d_{1}$ and $d_{2}$ distances (lying approximately along the respective $X_{1}$ and $X_{2}$ principal axes) with temperature is contrary compared to the heating run. Consequently, a weak NTE of $-4(2) \mathrm{MK}^{-1}$ is observed along $d_{2}$ on cooling in the $340-$ $100 \mathrm{~K}$ temperature range. The elasticity tensor calculations correctly showed which modes of SION-2 are soft and how it can deform, thus supporting its NTE behaviour. In addition, a uniaxial NLC effect is computationally predicted for this material.

The example of SION-2 can trigger further experimental and computational research efforts in this field, and so Ln-based MOFs endowed with mobile guest species entrapped in the structural voids can establish themselves as a novel unique class of materials exhibiting strong and tuneable NTE effects. 


\section{Acknowledgements}

KCS thanks the Swiss National Science Foundation (SNSF) for funding under the Ambizione Energy Grant no. PZENP2_166888 and the Materials' Revolution: Computational Design and Discovery of Novel Materials (MARVEL) - DD4.5. Access to the BM01 Swiss-Norwegian Beamline at the ESRF, Grenoble, France, is gratefully acknowledged. AG thanks Dr. Dmitry Chernyshov and Dr. lurii Dovgaliuk for fruitful discussions, and Ms Samantha L. Anderson for sample preparation. SMM thanks Dr. Gloria Capano for useful discussions.

\section{Conflicts of interest}

There are no conflicts to declare.

\section{References}

1. J. S. O. Evans, J. Chem. Soc., Dalton Trans., 1999, $3317-$ 3326.

2. (a) J. Chen, L. Hu, J. Deng and X. Xing, Chem. Soc. Rev., 2015, 44, 3522-3567; (b) Z. Liu, Q. Gao, J. Chen, J. Deng, K. Lin and X. Xing, Chem. Commun., 2018, 54, 5164-5176.

3. K. Takenaka, Sci. Technol. Adv. Mater., 2012, 13, 013001.

4. J. R. Salvador, F. Guo, T. Hogan and M. G. Kanatzidis, Nature, 2003, 425, 702-705.

5. (a) T. A. Mary, J. S. O. Evans, T. Vogt and A. W. Sleight, Science, 1996, 272, 90-92; (b) J. N. Hancock, C. Turpen, Z. Schlesinger, G. R. Kowach and A. P. Ramirez, Phys. Rev. Lett., 2004, 93, 225501.

6. B. K. Greve, K. L. Martin, P. L. Lee, P. J. Chupas, K. W. Chapman and A. P. Wilkinson, J. Am. Chem. Soc., 2010, 132, 15496-15498.

7. W. Yao, X. Jiang, R. Huang, W. Li, C. Huang, Z. Lin, L. Li and C. Chen, Chem. Commun., 2014, 50, 13499-13501.

8. L. Hu, J. Chen, L. Fan, Y. Ren, Y. Rong, Z. Pan, J. Deng, R. Yu and X. Xing, J. Am. Chem. Soc., 2014, 136, 13566-13569.

9. A. L. Goodwin and C. J. Kepert, Phys. Rev. B, 2005, 71, 140301.

10. K. Takenaka and H. Takagi, Appl. Phys. Lett., 2005, 87, 261902.

11. Y. Wu, A. Kobayashi, G. J. Halder, V. K. Peterson, K. W. Chapman, N. Lock, P. D. Southon and C. J. Kepert, Angew. Chem., Int. Ed., 2008, 47, 8929-8932.

12. Y. Wu, V. K. Peterson, E. Luks, T. A. Darwish and C. J. Kepert, Angew. Chem., 2014, 53, 5175-5178.

13. N. Lock, Y. Wu, M. Christensen, L. J. Cameron, V. K. Peterson, A. J. Bridgeman, C. J. Kepert and B. B. Iversen, J. Phys. Chem. C, 2010, 114, 16181-16186.

14 Z. Liu, Q. Li, H. Zhu, K. Lin, J. Deng, J. Chen and X. Xing, Chem. Commun., 2018, 54, 5712-5715.

15. (a) A. C. Bailey and B. Yates, J. Appl. Phys., 1970, 41, 50885091; (b) S. J. Hibble, A. M. Chippindale, A. H. Pohl and A. C. Hannon, Angew. Chem., Int. Ed., 2007, 46, 7116-7118. H. Takahashi and R. Tamura, CrystEngComm, 2015, 17, 8888-8896.

17. (a) G. Shirane, S. Hoshino and K. Suzuki, Phys. Rev., 1950, 80, 1105-1106; (b) G. A. Rossetti, J. P. Cline and A. Navrotsky, J. Mater. Res., 1998, 13, 3197-3206.
18. X. G. Zheng, H. Kubozono, H. Yamada, K. Kato, Y. Ishiwata and C. N. Xu, Nat. Nanotechnol., 2008, 3, 724.

19. S. Khmelevskyi, I. Turek and P. Mohn, Phys. Rev. Lett., 2003, 91, 037201.

20. (a) K. S. Knight, J. Miner. Petrol. Sci., 2014, 109, 118-124; (b) W. G. Marshall, R. H. Jones and K. S. Knight, CrystEngComm, 2018, 20, 3246-3250.

21. A. L. Goodwin, M. Calleja, M. J. Conterio, M. T. Dove, J. S. O. Evans, D. A. Keen, L. Peters and M. G. Tucker, Science, 2008, 319, 794-797.

22. A. D. Fortes, E. Suard and K. S. Knight, Science, 2011, 331, 742-746.

23. (a) L. D. DeVries, P. M. Barron, E. P. Hurley, C. Hu and W. Choe, J. Am. Chem. Soc., 2011, 133, 14848-14851; (b) I. E. Collings, M. G. Tucker, D. A. Keen and A. L. Goodwin, CrystEngComm, 2014, 16, 3498-3506; (c) J. Pang, C. Liu, Y. Huang, M. Wu, F. Jiang, D. Yuan, F. Hu, K. Su, G. Liu and M. Hong, Angew. Chem., Int. Ed., 2016, 55, 7478-7482; (d) Z. Liu, C. Liu, Q. Li, J. Chen and X. Xing, Phys. Chem. Chem. Phys., 2017, 19, 24436-24439.

24. W. Cai and A. Katrusiak, Nat. Commun., 2014, 5, 4337.

25. (a) A. L. Goodwin, K. W. Chapman and C. J. Kepert, J. Am. Chem. Soc., 2005, 127, 17980-17981; (b) I. Grobler, V. J. Smith, P. M. Bhatt, S. A. Herbert and L. J. Barbour, J. Am. Chem. Soc., 2013, 135, 6411-6414; (c) H. L. Zhou, R. B. Lin, C. T. He, Y. B. Zhang, N. Feng, Q. Wang, F. Deng, J. P. Zhang and X. M. Chen, Nat. Commun., 2013, 4, 2534; (d) H. L. Zhou, Y. B. Zhang, J. P. Zhang and X. M. Chen, Nat. Commun., 2015, 6, 6917.

26. A. E. Phillips, A. L. Goodwin, G. J. Halder, P. D. Southon and C. J. Kepert, Angew. Chem., Int. Ed., 2008, 120, 1396 1399.

27. Q. Gao, J. Chen, Q. Sun, D. Chang, Q. Huang, H. Wu, A. Sanson, R. Milazzo, H. Zhu, Q. Li, Z. Liu, J. Deng and X. Xing, Angew. Chem., Int. Ed., 2017, 56, 9023-9028.

28. I. de Pedro, A. García-Saiz, J. Dupont, P. Migowski, O. Vallcorba, J. Junquera, J. Rius and J. Rodríguez Fernández, Cryst. Growth Des., 2015, 15, 5207-5212.

$29 . \quad$ B. R. Hester, A. M. dos Santos, J. J. Molaison, J. C. Hancock and A. P. Wilkinson, J. Am. Chem. Soc., 2017, 139, 1328413287.

30. J. Chen, Q. Gao, A. Sanson, X. Jiang, Q. Huang, A. Carnera, C. G. Rodriguez, L. Olivi, L. Wang, L. Hu, K. Lin, Y. Ren, Z. Lin, C. Wang, L. Gu, J. Deng, J. P. Attfield and X. Xing, Nat. Commun., 2017, 8, 14441.

31. S. R. G. Balestra, R. Bueno-Perez, S. Hamad, D. Dubbeldam, A. R. Ruiz-Salvador and S. Calero, Chem. Mater., 2016, 28, 8296-8304.

32. (a) L. Sarkisov, R. L. Martin, M. Haranczyk and B. Smit, J. Am. Chem. Soc., 2014, 136, 2228-2231; (b) A. U. Ortiz, A. Boutin, A. H. Fuchs and F. X. Coudert, Phys. Rev. Lett. 2012, 109, 195502; (c) S. M. Moosavi, P. G. Boyd, L. Sarkisov and B. Smit, ACS Cent. Sci., 2018, 4, 832-839. 33. J.-C. G. Bünzli, J. Cooord. Chem., 2014, 67, 3706-3733.

34. (a) T.-M. Zhao, S. Chen, R. Shang, B.-W. Wang, Z.-M. Wang and S. Gao, Inorg. Chem., 2016, 55, 10075-10082; (b) Z. Zhang, X. Jiang, G. Feng, Z. Lin, B. Hu and W. Li, J. Solid State Chem., 2016, 233, 289-293.

35. S. L. Anderson, A. Gładysiak, P. G. Boyd, C. P. Ireland, P. Miéville, D. Tiana, B. Vlaisavljevich, P. Schouwink, W. van Beek, K. J. Gagnon, B. Smit and K. C. Stylianou, CrystEngComm, 2017, 19, 3407-3413. 
36. V. Dyadkin, P. Pattison, V. Dmitriev and D. Chernyshov, J. Synchrotron Rad., 2016, 23, 825-829.

37. CrysAlisPro, Rigaku Oxford Diffraction, 1.171.38.43 edn., 2015.

38. (a) G. Sheldrick, Acta Cryst. Sect. A, 2015, 71, 3-8; (b) G. Sheldrick, Acta Cryst. Sect. C, 2015, 71, 3-8.

39. O. V. Dolomanov, L. J. Bourhis, R. J. Gildea, J. A. K. Howard and H. Puschmann, J. Appl. Cryst., 2009, 42, 339-341.

40. A. Spek, Acta Cryst. Sect. C, 2015, 71, 9-18.

41. M. J. Cliffe and A. L. Goodwin, J. Appl. Cryst., 2012, 45, 1321-1329.

42. V. A. Blatov, A. P. Shevchenko and D. M. Proserpio, Cryst. Growth Des., 2014, 14, 3576-3586.

43. P. Giannozzi, S. Baroni, N. Bonini, M. Calandra, R. Car, C. Cavazzoni, D. Ceresoli, G. L. Chiarotti, M. Cococcioni, I. Dabo, A. Dal Corso, S. de Gironcoli, S. Fabris, G. Fratesi, R. Gebauer, U. Gerstmann, C. Gougoussis, A. Kokalj, M. Lazzeri, L. Martin-Samos, N. Marzari, F. Mauri, R. Mazzarello, S. Paolini, A. Pasquarello, L. Paulatto, C. Sbraccia, S. Scandolo, G. Sclauzero, A. P. Seitsonen, A. Smogunov, P. Umari and R. M. Wentzcovitch, J. Phys. Condens. Mat., 2009, 21, 395502.

44. R. Gaillac, P. Pullumbi and F.-X. Coudert, J. Phys. Condens. Mat., 2016, 28, 275201.

45. J. F. Nye, Physical properties of crystals. Their representation by tensors and matrices, Oxford Univerity Press, Oxford, 1957.

46. P. Lama, R. K. Das, V. J. Smith and L. J. Barbour, Chem. Commun., 2014, 50, 6464-6467.

47. (a) A. B. Cairns and A. L. Goodwin, Phys. Chem. Chem. Phys., 2015, 17, 20449-20465; (b) F. Trousselet, A. Boutin and F.-X. Coudert, Chem. Mater., 2015, 27, 4422-4430.

48. R. W. Munn, J. Phys. C Solid State, 1972, 5, 535-542.

49. (a) A. L. Goodwin, D. A. Keen and M. G. Tucker, Proc. Natl. Acad. Sci. U. S. A., 2008, 105, 18708-18713; (b) J. Kusz, M. Zubko, A. Fitch and P. Gütlich, Z. Kristallogr., 2011, 226, 576-584; (c) H. J. Shepherd, T. Palamarciuc, P. Rosa, P. Guionneau, G. Molnar, J. F. Letard and A. Bousseksou, Angew. Chem., Int. Ed., 2012, 51, 3910-3914; (d) J. M. Ogborn, I. E. Collings, S. A. Moggach, A. L. Thompson and A. L. Goodwin, Chem. Sci., 2012, 3, 3011-3017.

50. W. Cai, A. Gładysiak, M. Anioła, V. J. Smith, L. J. Barbour and A. Katrusiak, J. Am. Chem. Soc., 2015, 137, 9296-9301. 